\title{
Índice de sustentabilidad ambiental de unidades de producción de maíz amarillo en sistemas agrícolas del valle de Pativilca, Lima, Perú
}

\author{
Environmental sustainability index of yellow corn production \\ units in agricultural systems in the Pativilca valley, Lima, Peru
}

Fernando Carlos Bravo Martínez ${ }^{1 *}$, Percy Zorogastúa Cruz ${ }^{1}$, Rember Pinedo Taco ${ }^{1}$

\section{RESUMEN}

El objetivo de la investigación fue definir el valor de los indicadores ambientales y el índice general de sostenibilidad ambiental de las unidades de producción de maíz amarillo duro (MAD) en el valle de Pativilca, Perú. De una población finita de 360 productores de MAD del valle de Pativilca se aplicaron 73 encuestas con preguntas estructuradas en la dimensión ambiental. Para determinar el Coeficiente de Impacto Ambiental (EIQ) y el Impacto Ambiental (IE) se obtuvieron los valores de EIQ para el ingrediente activo de 12 pesticidas. Luego se calculó la proporción de uso en campo para establecer el valor de impacto ambiental en campo (EI). Para ello se multiplicó el EIQ por la dosis, el porcentaje del ingrediente activo y el número de aplicaciones de cada pesticida. Para definir los niveles de sostenibilidad ambiental se emplearon herramientas metodológicas de análisis multicriterio. Los datos se ordenaron en una escala de valor de 1 a 5: 1 es el valor de menor sostenibilidad y 5 el valor ideal de sostenibilidad. Se constató un IE ha ${ }^{-1}$ de 101,11 , lo que representa un elevado impacto negativo en la población y el medio ambiente debido a las prácticas inadecuadas en la gestión de agroquímicos, con serias repercusiones en la sostenibilidad de los sistemas agrícolas. El índice de sustentabilidad ambiental con 2,54 representa un nivel muy bajo en la escala establecida en el estudio de sustentabilidad, al hallarse por debajo del umbral mínimo aceptable de sostenibilidad. Los indicadores de menor contribución en la sustentabilidad de las unidades de producción de MAD en el valle de Pativilca fueron gestión y conservación de suelos, gestión de plagas y gestión de agua con 2,39; 2,32 y 2,90. Estos índices se encuentran en la escala de sustentabilidad en un rango crítico para el sistema de producción y, por consiguiente, tienen una baja contribución en la sostenibilidad ambiental.

Palabras clave: comisión de regantes, contaminación ambiental, resiliencia, plaguicida agrícola, sistemas de cultivo.

\begin{abstract}
The research was carried out to define the value of the environmental indicators, and the general index of environmental sustainability of the production units of hard yellow corn (MAD) in the Pativilca valley, Peru. 73 surveys with structured questions in the environmental dimension were applied. To determine the Environmental Impact Coefficient (EIQ) and the Environmental Impact (IE), the EIQ values were obtained for the active ingredient of 12 pesticides, then the proportion of use in the field was calculated to obtain the value of environmental impact in the field. To define the levels of environmental sustainability, multicriteria analysis methodological tools were used. The data were ordered on a value scale of 1 to 5; 1 being the least sustainable value and 5 the ideal sustainability value. An IE $h a^{-1}$ of 101.11 was found, a value that represents a high negative impact on the population and the environment due to inadequate practices in handling agrochemicals. The soil management and conservation, pest management and water management subindicators are the ones with the least contribution in the operation of agricultural systems, as a consequence, the environmental sustainability index with 2.54 presents a very low level in the sustainability scale established in the study by not reaching the minimum acceptable threshold of sustainability.
\end{abstract}

Keywords: irrigation commission, environmental pollution, resilience, agricultural pesticide, cultivation systems

\section{Introducción}

Actualmente la humanidad enfrenta una crisis alimentaria, económica, social, energética y ambiental (Sánchez-Morales et al., 2014). Esta última provocada por el deterioro de suelos, la sobreexplotación y destrucción de bosques y selvas, la contaminación del agua, la extracción masiva de agua del manto freático y el cambio climático global acelerado debido a las actividades antropogénicas contemporáneas. Este fenómeno tiene impactos ambientales y económicos negativos (BM, 2010).

\footnotetext{
$1 \quad$ Universidad Nacional Agraria La Molina (UNALM). Lima, Perú.

* Autor por correspondencia: 20120487@lamolina.edu.pe
} 
Según la FAO (2014), para el año 2050 la población mundial alcanzará los 9200 millones de habitantes. Por lo tanto, para alimentar a una población mundial cada vez más numerosa no hay más opción que intensificar la producción agrícola (FAO, 2011). El proceso de intensificación será mayor en los países en vías de desarrollo y, por ello, considerando los frágiles agroecosistemas, será necesario tomar medidas adecuadas para la gestión de recursos naturales (Altieri y Nichols, 2000). De hecho, se requiere intensificar la producción agrícola basada en sistemas agrícolas que ofrezcan a los productores y a la sociedad en general una variedad de beneficios socioeconómicos, ambientales y relacionados con la productividad (FAO, 2011).

Los productores de maíz amarillo duro (MAD), en su afán de incrementar los rendimientos para competir con el mercado internacional recurren en ocasiones al uso indiscriminado de plaguicidas y fertilizantes (MINAGRI, 2018). Según información del Departamento de Agricultura de los Estados Unidos (USDA) de febrero de 2019, se prevé que la producción mundial de maíz amarillo duro para la campaña 2018/2019 podría llegar a 1100 millones de toneladas, mayor en 2,2\% respecto al año 2017 (1 076 millones de toneladas) (MINAGRI, 2018).

En el Perú actualmente hay 2,16 millones de productores, de los cuales aproximadamente el $80 \%$ son pequeños productores con menos de 5 ha (INEI, 2013). Asimismo, la agricultura es de baja productividad y rentabilidad principalmente para agricultores de sistemas familiares (Pinedo et al., 2018).

De acuerdo a las estadísticas del MINAGRI (2014), el maíz amarillo duro es el segundo cultivo con mayor superficie de siembra dentro de los cultivos transitorios (326.532 ha) y su crecimiento se debe principalmente a la alta demanda de la agroindustria nacional de alimentos balanceados para la actividad avícola, porcícola y de animales de engorde.

En el caso del valle de Pativilca, los productores pueden sembrar durante todo el año (MINAGRI, 2018). El sistema de producción es del tipo convencional, con uso intensivo de maquinaria agrícola, fertilizantes minerales, plaguicidas agrícolas y sistema de riego por gravedad (INEI, 2013), sin considerar en la mayoría de los casos las buenas prácticas agrícolas (BPA) o estrategias de producción de menor impacto ambiental como la rotación de cultivos, cobertura vegetal, asociación y diversificación de cultivos (Pinedo et al., 2020)
De acuerdo a la FAO (2014), las BPA se refieren al conjunto de principios, normas y recomendaciones técnicas aplicables a la producción, procesamiento y transporte de alimentos, con la finalidad de asegurar la protección de la higiene, la salud humana y el medio ambiente, mediante métodos ecológicamente seguros y económicamente factibles, que se traducen en la obtención de productos alimenticios y no alimenticios más inocuos y saludables para el autoconsumo y el consumidor. Las BPA son un componente de competitividad, que permite al productor rural diferenciar su producto. El Reglamento de Inocuidad Agroalimentaria Decreto Supremo N ${ }^{\circ}$ 004-2011-AG señala en su artículo $14^{\circ}$, que los productores de alimentos agropecuarios primarios deberán implementar los lineamientos sobre Buenas Prácticas de Producción e Higiene que establezca el Servicio Nacional de Sanidad Agraria (SENASA). Al respecto, esta entidad sanitaria del Perú recomienda que los plaguicidas, fertilizantes, material de propagación y equipos estén separados entre sí, alejados de la vivienda e identificados con un letrero por tipo de insumo (SENASA, 2013).

Por lo expuesto se puede concluir que los sistemas de producción de MAD en el valle de Pativilca son del modelo convencional. Según Bolívar (2011), la agricultura convencional se caracteriza por su alta dependencia de insumos externos, y se basa en el paquete tecnológico de la denominada revolución verde, cuyos componentes provienen de la industria química, mecánica y biológica. La producción se destina principalmente para el mercado y hace uso excesivo de energéticos fósiles, razón por la que se considera de alta entropía. Estos modelos de producción pueden llegar a alcanzar niveles de sustentabilidad en la dimensión económica y social; sin embargo, en la dimensión ambiental normalmente no los logran (Pinedo et al., 2020). Por lo tanto, es necesario definir los niveles de sostenibilidad utilizando indicadores y sintetizando estas dimensiones para hallar los valores de sustentabilidad (Pinedo et al., 2020; Sarandón, 2002).

A pesar del consenso mundial para lograr la sustentabilidad, aún no existe un acuerdo en la forma de cuantificar para tomar decisiones prácticas que encaucen el desarrollo sustentable (Bolívar, 2011; Badii, 2004). En el caso de su evaluación en agroecosistemas se requiere transformar aspectos complejos en otros más claros, que permitan 
detectar tendencias a nivel de sistema, denominados índices (Castelán et al., 2014). Atributos de los agroecosistemas como la estabilidad y resiliencia contribuyen en la capacidad del agroecosistema para mantener el equilibrio dinámico y estable a través del tiempo, la capacidad de retornar a su estado de equilibrio ante perturbaciones graves y de mantener beneficios (Astier et al., 2008). Por lo tanto, requiere un abordaje holístico y sistémico (Andreoli y Tellarini, 2000). Desde este punto de vista multidisciplinario, queda claro que hablar de sustentabilidad y su medición es un proceso sumamente complejo (Badii, 2004).

Por ello, se planteó este trabajo con el objetivo de evaluar la sustentabilidad del agroecosistema maíz en sus dos formas de gestión de los recursos naturales (tradicional y en transición al agroindustrial), para proponer sistemas de producción más sustentables.

\section{Materiales y métodos}

La investigación se realizó en el valle de Pativilca, provincia de Barranca, Región Lima, ubicada entre los paralelos $10^{\circ} 00^{\prime}$ y $11^{\circ} 00^{\prime}$ de latitud sur y entre los meridianos $76^{\circ} 46^{\prime}$ y $77^{\circ} 57^{\prime}$ de longitud oeste (Figura 1). Esta zona presenta un clima templado y desértico. Respecto a la precipitación, registra un nivel muy escaso, aproximadamente $7 \mathrm{~mm} / \mathrm{año}$. El abastecimiento hídrico proviene del río Pativilca.

Se utilizó la escala temporal transversal, y por esta razón se consideraron los años 2009 y 2010. La escala espacial fue el valle de Pativilca (SánchezMorales et al., 2014). La etapa de investigación fue no experimental, descriptiva y explicativa. En esta fase se evaluó la sostenibilidad de las unidades o parcelas productoras de MAD en la zona central del valle de Pativilca. La delimitación del área de estudio fue producto de un análisis y diagnóstico territorial previo de la cuenca, el cual determinó la identificación de la unidad geográfica-económica de interés que resultó ser la cuenca media y baja del río Pativilca, constituida por un conjunto de condiciones homogéneas físicas, económicas y sociales (Ayora, 2015).

De una población de 360 productores de maíz amarillo duro se obtuvo una muestra irrestricta aleatoria $(n=73)$. Luego, de acuerdo al modelo de Pinedo et al. (2018), se seleccionó a los agricultores mediante muestreo sistemático de tres comisiones de regantes del valle de Pativilca.

\section{Evaluación del Coeficiente de Impacto Ambiental (EIQ) y el Impacto Ambiental (IE)}

Se utilizó información sobre el tipo de pesticida, las dosis empleadas y la frecuencia de aplicaciones (CIP, 2009). El coeficiente de impacto ambiental (EIQ por sus siglas en inglés) y el impacto ambiental (EI) fueron desarrollados por la Universidad de Cornell, Estados Unidos. El impacto ambiental (EI por sus siglas en inglés) es un indicador que sirve para valorar el riesgo potencial resultante del uso de los pesticidas en cultivos agrícolas, tanto para los agricultores que los aplican como para los consumidores y el componente ecológico (CIP, 2009). El cálculo del EIQ se basó en una metodología de ponderación para 12 tipos de pesticida para el control de cinco plagas más importantes utilizados por los productores de MAD del valle de Pativilca, como se muestra en la siguiente relación matemática: EIQ $=(\mathrm{C}[(\mathrm{DT} * 5)$ $+(\mathrm{DT} * \mathrm{P})]+(\mathrm{C} \times[(\mathrm{S}+\mathrm{P}) / 2] * \mathrm{SY})+(\mathrm{L})+(\mathrm{F} * \mathrm{R})$
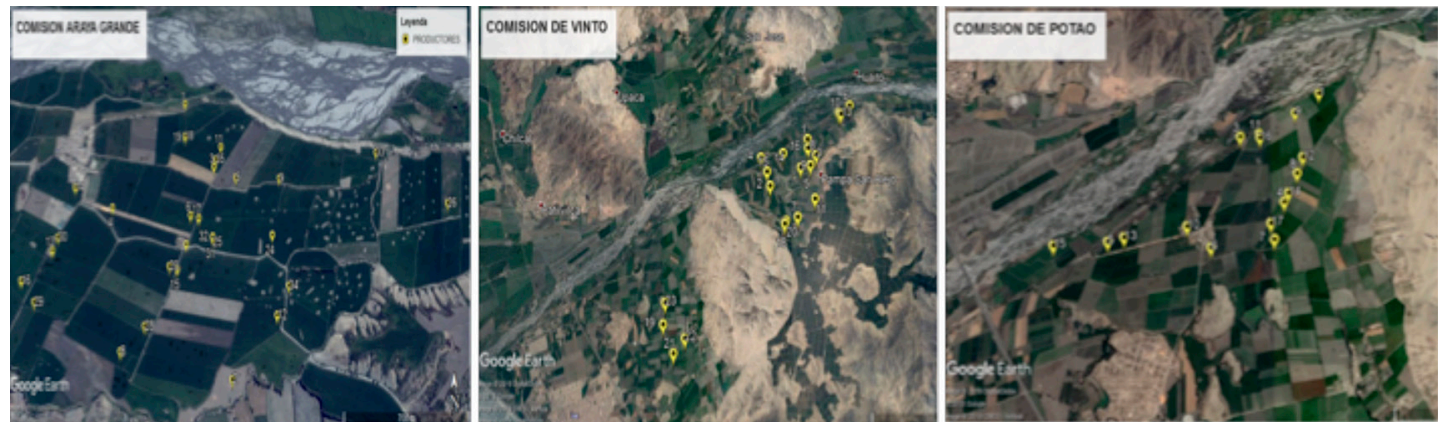

Figura 1. Ámbito de estudio de sostenibilidad de sistemas agrícolas de MAD en las comisiones de riego de Araya Grande, Vinto y Potao. Google Earth. 
$+(\mathrm{D} *[(\mathrm{~S}+\mathrm{P}) / 2] * 3)+(\mathrm{Z} * \mathrm{P} * 3)+(\mathrm{B} * \mathrm{P} * 5))$ 13. Donde $\mathrm{C}=$ Toxicidad crónica; $\mathrm{DT}=$ Toxicidad dermal; $\mathrm{P}=$ Vida media de residuos en superficie de planta; $\mathrm{S}=$ Vida media de residuos en el suelo; $\mathrm{SY}$ $=$ Sistematicidad $\mathrm{L}=$ Potencial de lixiviación $; \mathrm{F}=$ Toxicidad en peces; $\mathrm{R}=$ Potencial de escorrentía; $\mathrm{D}=$ Toxicidad en aves; $\mathrm{Z}=$ Toxicidad en abejas; $\mathrm{B}=$ Toxicidad en artrópodos benéficos. Una vez obtenidos los valores de EIQ para el ingrediente activo de cada pesticida, se calculó la proporción de uso en campo para obtener el valor de impacto ambiental en campo (EI). Para ello se multiplicó el EIQ por la dosis, el porcentaje del ingrediente activo y el número de aplicaciones de cada pesticida. A mayor valor del EI, mayor potencial de impacto ambiental negativo.

\section{Evaluación de sustentabilidad ambiental de la producción de MAD con uso de indicadores}

La evaluación se realizó con el uso de indicadores elaborados para la dimensión ambiental y de forma comparativa entre las tres comisiones de regantes a las que pertenece cada sector y que han servido para determinar los niveles de sostenibilidad de las unidades de producción de maíz amarillo duro (UPMAD) en el ámbito de estudio. La investigación se hizo aplicando el enfoque cualitativo (Flores y Sarandón, 2006) y la propuesta metodológica de análisis multivariado de Sarandón (2002), basada en la utilización de indicadores ambientales.

\section{Construcción de indicadores}

El desarrollo y uso de indicadores es una herramienta adecuada y flexible para evaluar tendencias, establecer diferencias entre fincas y detectar los puntos críticos de gestión de recursos para el logro de una agricultura sustentable (Sarandón y Flores, 2014). Se eligieron cuatro indicadores: gestión y conservación de suelos; gestión de plagas; gestión del agua (Tabla 1). Se trató de que los indicadores sean en lo posible fáciles de obtener e interpretar, que brinden la

Tabla 1. Indicadores de sostenibilidad ambiental de un sistema productivo de MAD.

\begin{tabular}{|c|c|c|c|}
\hline Dimensión & Indicador & Subindicador & Escalas de valor de los indicadores \\
\hline \multirow{9}{*}{ Ambiental } & \multirow{4}{*}{$\begin{array}{l}\text { A.- Conservación } \\
\text { de la vida suelo }\end{array}$} & A1.- Rotación de cultivos. & $\begin{array}{l}\text { (5) Siembra MAD y descansa el terreno; (4)Siembra MAD y } 1 \\
\text { campaña páprika; (3) Siembra MAD, paprika y MAD; (2) Siembra } \\
\text { MAD dos campañas; (1) Siembra MAD tres campañas. }\end{array}$ \\
\hline & & $\begin{array}{l}\text { A2.- Niveles y tipos de } \\
\text { fertilización. }\end{array}$ & $\begin{array}{l}\text { (5) Orgánico procesado; (4)Orgánico sin procesar; (3) químico } \\
\text { y alta incorporación de MO; (2) Químico y baja incorporación } \\
\text { de MO (1) Solo químico. }\end{array}$ \\
\hline & & $\begin{array}{l}\text { A3.- Incorporación de } \\
\text { materia orgánica. }\end{array}$ & $\begin{array}{l}(5)>\text { a } 10,1 \mathrm{tha}^{-1}(4) \text { de } 7,1 \text { a } 10 \mathrm{tha}^{-1}(3) \text { de } 5,1 \text { a } 7 \mathrm{tha}^{-1} ;(2) \mathrm{de} \\
3,1 \text { a } 5 \mathrm{tha}^{-1} \text {; (1) Menor a } 3 \mathrm{tha}^{-1} \text {. }\end{array}$ \\
\hline & & $\begin{array}{l}\text { A4.- gestión residuos de } \\
\text { cosecha. }\end{array}$ & $\begin{array}{l}\text { (5) Deja en parcela como cobertura (4) Procesa compost (3) Recoge } \\
\text { para alimento de ganado; (2) lo vende (1) quema. }\end{array}$ \\
\hline & \multirow{3}{*}{$\begin{array}{l}\text { B.- Gestión de } \\
\text { plagas }\end{array}$} & B1.- Barreras vivas. & $\begin{array}{l}\text { (5) Tiene cercos, barreras y cultivos refugio ; (4)Tiene barreras y } \\
\text { zonas de refugio en un solo lado; (3) Siembra de barreras vivas } \\
\text { como cortavientos; (2) Solo cuenta con cercos vivos (1) No aplica } \\
\text { esta práctica por desconocimiento. }\end{array}$ \\
\hline & & $\begin{array}{l}\text { B2.- Método de control } \\
\text { de plagas. }\end{array}$ & $\begin{array}{l}\text { (5) MIP (4) Labores culturales y bioplaguicidas; (3) Químico, } \\
\text { labor cultural y bioplaguicidas; (2) } 3 \text { Químico y algunas labores } \\
\text { culturales; (1) Químico. }\end{array}$ \\
\hline & & $\begin{array}{l}\text { B3.- Frecuencia de apli- } \\
\text { cación plaguicidas. }\end{array}$ & $\begin{array}{l}\text { 5) de } 1 \text { a } 2 \text { aplicaciones; 4) de } 3 \text { a } 4 \text { aplicaciones; } 3 \text { ) de } 5 \text { a } 6 \\
\text { aplicaciones; } 2 \text { ) de } 7 \text { a } 8 \text { aplicaciones; } 1 \text { ) más de } 8 \text { aplicaciones. }\end{array}$ \\
\hline & \multirow[t]{2}{*}{$\begin{array}{l}\text { C.- Gestión de } \\
\text { agua }\end{array}$} & C1.- Sistema de riego. & $\begin{array}{l}\text { (5) Fertirriego; (4) Riego por goteo (3) Riego por gravedad } \\
\text { con regantes expertos; (2) Riego por gravedad con regantes } \\
\text { semiexpertos (1) Riego por gravedad con regantes inexpertos. }\end{array}$ \\
\hline & & C2.- Calidad del agua. & 5) muy buena; 4) buena; 3) regular); 2) mala; 1 ; muy mala. \\
\hline
\end{tabular}


información necesaria y que permitan detectar tendencias en el ámbito de estudio (Pinedo et al., 2017; Sarandón, 2002; Sarandón et al. 2006).

Para comparar las unidades de producción (parcelas) y facilitar el análisis de los indicadores y subindicadores identificados para la dimensión social, se estandarizaron los datos modificando la propuesta original de Sarandón (2002) de 0 a 4 a una escala de 1 a 5 como lo realizado por Pinedo et al. (2018) y Pinedo et al. (2020). En la escala el valor 1 se considera como valor crítico de menor aporte a la sustentabilidad de las UPMAD; el valor 3 umbral mínimo a partir del cual todo indicador puede ser considerado sustentable y 5 el umbral o valor máximo de sostenibilidad (Pinedo et al., 2018). Esto facilitó la integración de varios indicadores de distinta naturaleza en otros más sintéticos o robustos (Sarandón, 2002). La utilización de razones matemáticas ha permitido estandarizar los valores absolutos y optimizar la gestión de los datos, además de una mejor comprensión de la dinámica propia de los indicadores calculados (Pinedo et al., 2020; Sarandón, 2002).

Posteriormente, para determinar el Indicador Ambiental (IA), los subindicadores de cada indicador fueron ponderados con la siguiente relación matemática: $\mathrm{IA}=[(2 * \mathrm{~A} 1+2 * \mathrm{~A} 2+\mathrm{A} 3+$ $\mathrm{A} 4) / 6+(2 * \mathrm{~B} 1+2 * \mathrm{~B} 2+\mathrm{B} 3) / 5+2(\mathrm{C} 1+\mathrm{C} 2) / 2)] / 3$. Los indicadores fueron ponderados multiplicando el valor de la escala por un coeficiente de acuerdo a la importancia relativa de cada variable respecto a la sustentabilidad. Este coeficiente multiplica tanto el valor de las variables que forman el indicador, como el de los indicadores, para construir indicadores de mayor nivel o índices (Pinedo et al., 2020). La validación de la ponderación de cada subindicador fue por consenso entre técnicos, expertos y agricultores (Pinedo et al., 2017).

\section{Resultados y discusión}

\section{Evaluación del Coeficiente de Impacto Ambiental (EIQ) y el Impacto Ambiental (IE)}

Los resultados muestran que el IE ha- ${ }^{-1}$ es de 101,11 , valor que representa un elevado impacto ambiental negativo en campo, es decir, existe un alto riesgo causado por los pesticidas utilizados para el control de plagas y enfermedades en el cultivo de maíz amarillo duro, tanto para el ambiente como para las personas. Estos riesgos además comprometen la sostenibilidad de los sistemas agrícolas (Tabla 2). Ello se explica porque de los 12 plaguicidas usados por los agricultores y de manera muy frecuente, el $25 \%$ pertenece a aquellos que son extremadamente peligrosos (clasificados como "muy tóxicos"), el $42 \%$ corresponde a aquellos que son moderadamente peligrosos (clasificados como "nocivos") y $33 \%$ son los "ligeramente peligrosos" (clasificados como insecticidas "de cuidado"). Todos estos insecticidas pertenecen a las categorías I (etiqueta roja), II (etiqueta amarilla) y III (etiqueta azul). No utilizan ninguno de categoría IV (etiqueta verde). Con respecto a la frecuencia de aplicación de plaguicidas, Rodríguez (2018) menciona que en barranca en el cultivo de maíz se presentan plagas como Agrotis spp., Spodopthera frugiperda, Heliothis zea y el complejo de la mancha alquitrán (causado por la interacción entre Phyllachora maydis, Monographella maydis y Coniothyrium phyllachorae). Para controlar estas poblaciones de plagas se realizan hasta 14 aplicaciones.

Según Bejarano (2017), los pesticidas muy tóxicos generan toxicidad alta en abejas, toxicidad crónica y aguda alta en humanos, permanecen mucho tiempo en el ambiente (persistentes en el agua, suelo, sedimentos), se desplazan a grandes distancias y son bioacumulables.

A esto se suma la gestión inadecuada de los envases de los pesticidas químicos, pues según los resultados de la encuesta, el 54,7\% reporta que estos envases son dejados en la chacra y un $24 \%$ indica que los arrojan a canales de regadío, ríos o acequias. Esto genera una mayor contaminación al ambiente o al agua. Sólo un tercio de los agricultores manifiestan que los envases son depositados en un almacén temporal. Los envases de productos químicos vacíos deben ser manejados de manera que se eviten riesgos para la salud humana y el medio ambiente.

Otro factor que se suma a la generación de una mayor contaminación ambiental es el poco respeto que tienen los agricultores a las indicaciones de uso de cada pesticida. De esta manera, el 46,7\% de ellos indican que usan más de la dosis recomendada en sus aplicaciones. Esto lo hacen con la finalidad de asegurar el efecto del pesticida. Tal como lo reporta Harris (2000), el incremento en las dosis y la gestión inadecuada de los plaguicidas son causa de acumulación de residuos de agroquímicos en diversos ecosistemas, problemas en la salud 
Tabla 2. Coeficiente de Impacto Ambiental (EIQ) y el Impacto Ambiental (IE)

con base en los ingredientes activos utilizados en sistemas agrícolas de maíz amarillo duro.

\begin{tabular}{|c|c|c|c|c|c|}
\hline \multirow{2}{*}{ Ingrediente activo } & \multirow{2}{*}{ Concentración } & \multirow{2}{*}{ Cantidad/ha } & \multirow{2}{*}{$\frac{\text { Aplicaciones en verano }}{\left(\mathrm{N}^{\mathrm{o}}\right)}$} & \multirow{2}{*}{ EIQ } & \multirow{2}{*}{ EI t ha- } \\
\hline & & & & & \\
\hline \multicolumn{6}{|l|}{ Gusano de tierra } \\
\hline Clorpyrifos & 0,48 & 0,4 & 1 & 43,5 & 8,35 \\
\hline Metamidofos & 0,6 & 0,7 & 1 & 36,8 & 15,46 \\
\hline Carbofuran & 0,48 & 0,5 & 1 & 50,67 & 12,16 \\
\hline Metomil & 0,2 & 0,2 & 2 & 30,7 & 2,46 \\
\hline & & & \multicolumn{2}{|l|}{ Total } & 3,42 \\
\hline \multicolumn{6}{|l|}{ Gusano cogollero } \\
\hline Spinetoram & 0,06 & 0,1 & 4 & 17,7 & 0,42 \\
\hline Metomil & 0,2 & 0,2 & 5 & 30,7 & 6,14 \\
\hline Clorpyrifos & 0,48 & 0,4 & 1 & 43,5 & 8,35 \\
\hline Chlorantraniliprole & 0,18 & 0,1 & 2 & & \\
\hline & & & \multicolumn{2}{|l|}{ Total } & 14,92 \\
\hline \multicolumn{6}{|l|}{ Chinche } \\
\hline Imidacloprid & 0,7 & 0,2 & 2 & 34,9 & 9,77 \\
\hline Dimetoato & 0,4 & 0,5 & 2 & 74 & 29,6 \\
\hline & & & \multicolumn{2}{|l|}{ Total } & 39,37 \\
\hline \multicolumn{6}{|l|}{ Mazorquero } \\
\hline Cypermetrina & 0,2 & 0,2 & 2 & 27,3 & 2,18 \\
\hline Chlorantraniliprole & 0,18 & 0,1 & 3 & & \\
\hline Lufernuron & 0,05 & 0,2 & 2 & 25,33 & 0,51 \\
\hline & & & \multicolumn{2}{|l|}{ Total } & 2,69 \\
\hline \multicolumn{6}{|l|}{ Mancha de asfalto } \\
\hline Tebuconazole & 0,25 & 0,2 & 2 & 40,3 & 4,03 \\
\hline Pyraclostrobin+Epoxiconazol & 0,133 & 0,2 & 2 & 31,45 & 1,67 \\
\hline & & & \multicolumn{2}{|l|}{ Total } & 5,7 \\
\hline & & & & & 101,11 \\
\hline
\end{tabular}

humana, daños al medio ambiente, resistencia de los insectos a insecticidas y resurgencia de plagas, incremento de plagas secundarias y disminución de enemigos naturales. Estos son aspectos a tener muy en cuenta. En contraste, el 45,3\% reporta que sí usa la dosis indicada.

La salud de los agricultores se ve afectada porque usan indumentarias inadecuadas para las labores de fumigación. Se constató que el $56 \%$ utiliza polos viejos, seguido de quienes usan un simple gorro de tela. Mientras que un poco más de un tercio utiliza botas de jebe y el $24 \%$ usa alguna mascarilla para protegerse la cara. Muy pocos utilizan guantes o lentes. Hay que resaltar que esta vestimenta no cumple los criterios establecidos para una buena protección del agricultor. De esta manera, el grado de exposición y contacto directo con el pesticida es mucho mayor, con las consecuencias negativas que esto conlleva. Según Corra (2009), las malas prácticas agrícolas, así como la falta de capacitación del personal que manipula los plaguicidas y la escasez de elementos de protección constituyen un agravante de la problemática de contaminación del suelo con plaguicidas. Al respecto, el Servicio Nacional de Sanidad Agraria del Perú recomienda que los plaguicidas, fertilizantes, material de propagación y equipos estén separados entre sí, alejados de la vivienda e identificados con un letrero por tipo de insumo (SENASA, 2013).

Un impacto mayor se origina en la contaminación del suelo como consecuencia del destino que se les da a los residuos procedentes del lavado de envases. En esa línea, el siguiente cuadro reporta que 74,7\% 
de los agricultores, luego de lavar los envases de los pesticidas los arrojan al suelo. El 18,7\% contamina el agua arrojando estos vertimientos a los canales de regadío, al río o a las acequias. Muy pocos entierran los envases. Al respecto, Del Puerto et al. (2014) indican que los plaguicidas se incorporan a las aguas por descargas provenientes del lavado de equipos empleados en la mezcla y aplicación de dichos productos. En ambos casos están afectando a dos recursos naturales importantes como son el suelo y el agua, lo que genera una contaminación generalizada al ambiente.

\section{Evaluación de sostenibilidad ambiental con uso de indicadores}

De acuerdo a la ponderación establecida en el estudio y la propuesta de análisis multicriterio de Sarandón (2002) y Sarandón y Flores (2014), el indicador gestión y conservación de suelos resultó con un valor de 2,39. Por lo tanto, se considera como un valor no sustentable por ubicarse por debajo del umbral mínimo de sostenibilidad (UMS). Según Pinedo et al. (2018), para ser sostenible este valor debe ser mayor a 3. Asimismo, el indicador de gestión de plagas presenta un valor no sustentable $(2,32)$. Este indicador es el valor más bajo comparado con los indicadores de gestión y conservación de suelos, así como gestión del agua (Figura 2). Los subindicadores críticos de mayor influencia fueron el plan de rotación de cultivos, intensidad de labranza de suelos, cantidad de materia orgánica incorporada a los suelos y el tratamiento o destino de los residuos de cosecha. Los sistemas de producción convencional son altamente dependientes de insumos externos, usan fertilizantes, maquinaria y equipo en forma intensiva y normalmente descuidan el recurso suelo (Altieri y Nicholls, 2000; Pinedo et al., 2018; Bolívar, 2011).

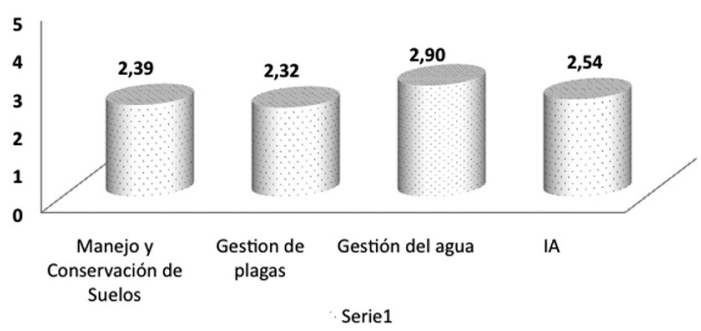

Figura 2. Valor de los indicadores e índice ambiental en las unidades de producción de maíz amarillo duro en Pativilca, Perú.
Con relación a los indicadores de gestión de plagas que resultó con un valor de 2,32 y gestión del agua con 2,90, igualmente ambos son valores menores a 3. Por lo tanto, se encuentran en la condición crítica de sustentabilidad de acuerdo a la escala establecida por Pinedo et al. (2018). Al respecto, Sarandón (2002) se refiere a la agricultura sostenible como aquella que permite mantener en el tiempo un flujo de bienes y servicios que satisfacen las necesidades socioeconómicas y culturales de la población, dentro de los límites biofísicos que establece el correcto funcionamiento de los sistemas naturales (agroecosistemas) que lo soportan.

En el análisis de sustentabilidad de las unidades de producción de MAD a nivel de comisiones de regantes, los tres grupos: Araya Grande, Potao y Vinto resultaron no sustentables con valores de $2,69,2,54$ y 2,43 respectivamente (Figura 3 ). Las tres comisiones se encuentran en una condición crítica cuyos valores menores al UMS reflejan un deterioro del recurso suelo por el uso intensivo de suelos, aplicación de altas dosis de fertilizantes y pesticidas agrícolas, así como inadecuadas prácticas de riego y gestión del agua (Altieri y Nichols, 2000; Sarandón y Flores, 2014).

Por ejemplo, sobre la intensificación de uso de suelos, en la zona de Barranca, en el cultivo de maíz, se registran hasta siete operaciones mecánicas de labranza (un pase de arado, de rastra, el surcado para la siembra y tres aporques para la incorporación de fertilizantes). Al respecto, el SENASA (2013), como parte de las BPA recomienda que durante la preparación del terreno se implementen sistemas de labranza mínima para conservar el suelo evitando la erosión y la compactación.

En el análisis a nivel de subindicadores y el aporte que brindan a cada indicador (Figura 4),

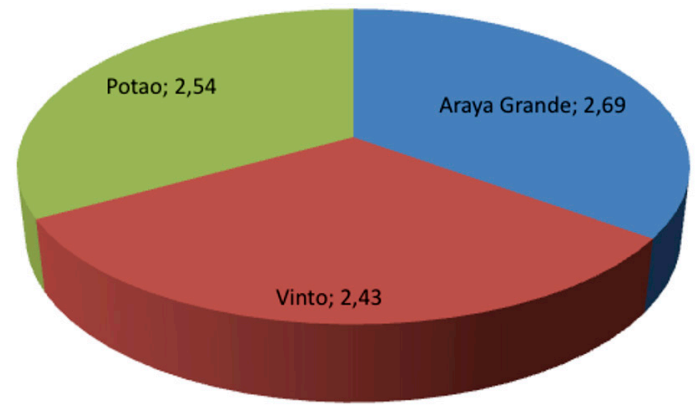

Figura 3. Indicadores ambientales por comisiones de regantes de producción de MAD en el valle de Pativilca, Perú. 


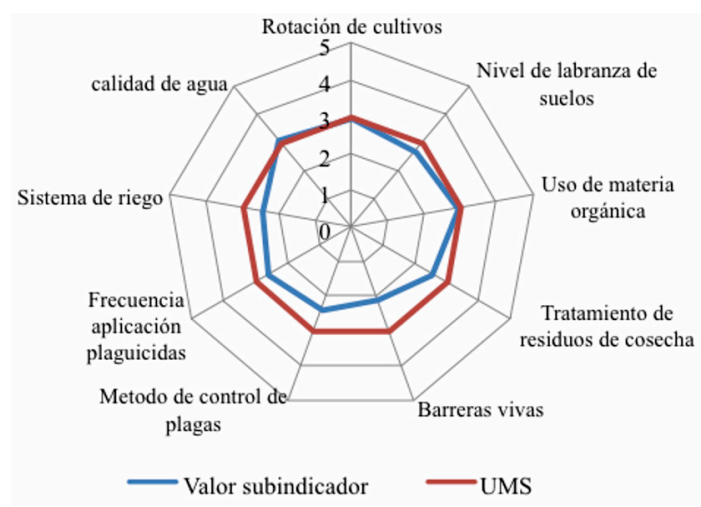

Figura 4. Representación tipo ameba de los indicadores y subindicadores de sostenibilidad ambiental en las unidades agrícolas de producción de MAD, Pativilca, Perú. UMS = umbral mínimo de sostenibilidad.

en la Figura tipo ameba se observa que solo el subindicador de calidad de agua se encuentra con un valor ligeramente superior al UMS, pero se ubica en una escala de sustentabilidad débil (Pinedo et al., 2018). Los subindicadores de rotación de cultivos y uso de materia orgánica se aproximan al UMS; sin embargo, se ubican en la escala de sustentabilidad crítica. Por lo tanto, prácticas consecutivas de monocultivo y uso inadecuado e ineficiente de los recursos agua y suelo pueden afectar esa condición temporal hasta niveles de sustentabilidad de riesgo extremo
(Pinedo et al., 2018). Para mantenerse en un nivel aceptable de sustentabilidad los agroecosistemas y subsistemas de cultivo requieren prácticas que no rebasen sus límites biofísicos (Sarandón, 2002). Ambos recursos extremadamente sensibles pueden ser afectados por modelos de producción intensivos y con enfoque solamente productivista (Bolívar, 2011; Sarandón y Flores, 2014; Altieri y Nichols, 2000).

\section{Conclusiones}

El indicador ecológico (IE ha-1) es de 101,11, valor que representa un elevado impacto ambiental negativo en campo, es decir, existe un alto riesgo causado por los pesticidas utilizados para el control de plagas y enfermedades en el cultivo de maíz amarillo duro, tanto para el ambiente como para las personas. Estos riesgos además comprometen la sostenibilidad de los sistemas agrícolas.

Con respecto al análisis de sustentabilidad utilizando indicadores, el indicador ambiental (IA) resulta con un nivel de sustentabilidad baja. Los indicadores de gestión y conservación de suelos, gestión de plagas y gestión de agua, con valores de 2,39, 2,32 y 2,90, se encuentran en la escala de sustentabilidad crítica. Por consiguiente, no contribuyen positivamente en la sustentabilidad de las unidades de producción de maíz amarillo duro (MAD) en el valle de Pativilca.

\section{Literatura Citada}

Altieri, M.; Nicholls, C.

2000. Agroecología. Teoría y práctica para una agricultura sustentable. Primera edición. PNUMA. D.F., México.

Andreoli, M.; Tellarini, V.

2000. Farm sustainability evaluation: methodology and practice. Agriculture, Ecosystems and Environment, 77: 43-52.

Astier, M.; Masera, O.; Galván, Miyoshi, Y.

2008. Evaluación de la sustentabilidad. Un enfoque dinámico y multidimensional. Valencia, España. 200 p.

Badii, M.H.

2004. Desarrollo sustentable: fundamentos, perspectivas y limitaciones. Innovaciones de Negocios, 1(2): 199-227.

Bejarano, F.

2017. Los plaguicidas altamente peligrosos en México. Red de Acción sobre plaguicidas y alternativas en México, A. BM. C. (RAPAM). México, 351 p.

2010. Informe sobre desarrollo mundial 2010: desarrollo y cambio climático. Banco Mundial. Nueva York. 60 p.
Bolívar, $\mathrm{H}$.

2011. Metodologías e indicadores de evaluación de sistemas agrícolas hacia el desarrollo sostenible. CICAG., 8 (1): 1-18.

Castelán, R.; Tamariz, T.; Ruiz, J.; Linares, G.

2014. Evaluación de la sustentabilidad de la actividad agrícola de tres localidades campesinas en Pahuatlán, Puebla. Ecosistemas y Recursos Agropecuarios, 1(3): 219-231.

CIP.

2009. Guía introductoria para la evaluación de impactos en programas de manejo integrado de plagas (MIP). Centro Internacional de la Papa. Lima, Perú. 44 p.

Corra, L.

2009. Herramientas de capacitación para el manejo responsable de plaguicidas y sus envases: efectos sobre la salud y prevención de la exposición. Organización Panamericana de la Salud (OPS). Buenos Aires, Argentina. 300 p.

Del Puerto, A; Suárez, S.; Palacio, D.E.

2014. Efectos de los plaguicidas sobre el ambiente y la salud. Rev. Cubana Hig Epidemiol, 52 (3): 372-387. 
FAO.

2011. Ahorrar para crecer. Guía para los responsables de las políticas de intensificación sostenible de la producción agrícola en pequeña escala (ISPA). Organización de las Naciones Unidas para la Alimentación y la Agricultura. Roma, Italia. 102 p.

FAO.

2014. Buenas Prácticas Agrícolas (BPA): en busca de sostenibilidad, competitividad y seguridad alimentaria. Organización de las Naciones Unidas para la Agricultura y la Alimentación. Roma, Italia. 699 p.

Harris, J.

2000. Chemical Pesticide Markets, Health Risks and Residues.

INEI. Ascot, UK: CABI Bioscience. 90 p.

2013. Resultados definitivos. IV Censo Nacional Agropecuario 2012. Instituto Nacional de Estadística e Informática. Perú. $62 \mathrm{p}$.

MINAGRI (Ministerio de Agricultura y Riego, Perú).

2014. Intenciones de siembra. Campaña agrícola agosto 2013 - julio 2014. Oficina de Estudios Económicos y Estadísticos (OEEE-MINAGRI). 595 p.

MINAGRI.

2018. Plan Nacional de cultivos. Campaña agrícola 2019-2020. Ministerio de Agricultura y Riego. Lima, Perú. 322 p.

Pinedo, R.; Gómez, L.: Julca, A.

2017. Caracterización de sistemas de producción de quinua (Chenopodium quinoa Willd.) en el distrito de Chiara, Ayacucho. Aporte santiaguino, 10(2): 351-364.

Pinedo, R.; Gómez, L.; Julca, A.

2018. Sostenibilidad de sistemas de producción de quinua (Chenopodium quinoa Willd.). Ecosistemas y Recursos Agropecuarios, 5(15): 399-409.
Pinedo, R.; Gómez, L.; Julca, A.

2018. Sustentabilidad de sistemas de producción de quinua (Chenopodium quinoa). Ecosistemas y Recursos Agropecuarios, 5(15): 399-409.

Pinedo-Taco, R.; Gómez-Pando, L.; Julca-Otiniano, A. 2020. Sostenibilidad ambiental de la producción de quinua (Chenopodium quinoa Willd.) en los valles interandinos del Perú. Ciencia y Tecnología Agropecuaria, 21(3): e1309.

Sánchez-Morales, P.; Ocampo-Fletes, I.; Parra-Inzunza, F.; Sánchez-Escudero, J.; María-Ramírez, A.; ArgumedoMacías, A.

2014. Evaluación de la sustentabilidad del agroecosistema maíz en la región de Huamantla, Tlaxcala, México. Agroecología, (9): 111-122.

Sarandón, S.; Zuluaga, M.; Cieza, R.; Gómez, C.; Janjetic, L.; Negrete, E.

2006. Evaluación de la sustentabilidad de sistemas agrícolas en fincas de Misiones, Argentina, mediante el uso de indicadores. Agroecología, 1: 19-28.

Sarandón, S.J.

2002. El desarrollo y uso de indicadores para evaluar la sustentabilidad de los agroecosistemas. En Agroecología: el camino hacia una agricultura sustentable. Ediciones Científicas Americanas, (20): 393-414.

Sarandón, S.J.; Flores, C.

2014. Agroecología: bases teóricas para el diseño y manejo de agroecosistemas sustentables. Argentina. Buenos Aires. $466 \mathrm{p}$.

SENASA.

2013. Guía de Buenas Prácticas Agrícolas. Servicio Nacional de Sanidad Agraria. Ministerio de Agricultura y Riego. Lima, Perú. 25 p. 
